\title{
Manejo anestésico en neonatos con tumoraciones pulmonares
}

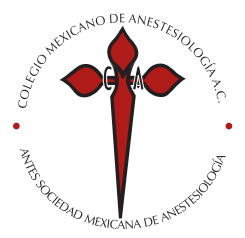

\section{Anesthetic management in neonates with lung tumors}

\author{
Dr. Fabio Andrés Avellaneda-Suárez,* \\ Dr. Edgardo Luis Vizcaíno-Ramírez, ${ }^{\ddagger}$ Dr. Juan Carlos Ramírez-Mora ${ }^{\S}$
}

Citar como: Avellaneda-Suárez FA, Vizcaíno-Ramírez EL, Ramírez-Mora JC. Manejo anestésico en neonatos con tumoraciones pulmonares. Rev Mex Anestesiol. 2022; 45 (2): 138-141. https://dx.doi.org/10.35366/103890

\begin{abstract}
RESUMEN. Introducción: Los tumores pulmonares congénitos son patologías poco frecuentes; asimismo, cuando requieren intervención quirúrgica, el médico anestesiólogo se enfrenta a un reto en el manejo de estos pacientes. Presentación del caso: En este artículo describimos el caso y el manejo anestésico de un recién nacido de 37.5 semanas de gestación (SDG) y 26 días de vida extrauterina, programado para toracotomía posterolateral izquierda más lobectomía inferior izquierda y colocación de catéter venoso central bajo anestesia combinada (anestesia general balanceada más bloqueo caudal con bupivacaína y morfina). Conclusiones: Las consideraciones anestésicas para los procedimientos quirúrgicos en neonatos y/o pacientes pediátricos se convierten en un reto para el anestesiólogo, debido a la inmadurez de algunos de los sistemas, se considera que la técnica anestésica idónea para el adecuado manejo de los pacientes neonatales sigue siendo la técnica combinada. El anestesiólogo debe mantener en mente dicha técnica así como los eventos y/o efectos secundarios que se puedan derivar de la misma y de este modo instaurar de manera oportuna el tratamiento pertinente.
\end{abstract}

ABSTRACT. Introduction: Congenital lung tumors are rare pathologies, likewise when surgical intervention is required, the anesthesiologist faces a challenge in the anesthetic management of these patients. Presentation of the case: The following article describes the case of a 26-day-old newborn child scheduled for left posterolateral thoracotomy plus lower left lobectomy and central venous catheter placement under combined anesthesia (balanced general anesthesia plus caudal block with bupivacaine and morphine). Conclusions: Anesthetic considerations for surgical procedures in neonates and/or pediatric patients become a challenge for the anesthesiologist, due to the immaturity of some of the systems, thus considering that the ideal anesthetic technique for the proper management of in neonatal patients, the combined technique continues, with epidural blocks for the management of peri- and postoperative pain. The anesthesiologist must keep in mind this technique as well as the events and/or side effects that may derive from it, thus establishing the pertinent treatment in a timely manner.

\begin{abstract}
Palabras clave: Tumores pulmonares, bloqueo caudal, anestesia combinada, neonato.
\end{abstract}

Keywords:

Lung tumors, caudal block, combined anesthesia, neonate.

\begin{abstract}
* Residente de Tercer año de Anestesiología.

‡ Residente de Primer año de Anestesiología.

${ }^{\S}$ Médico adscrito al Servicio de Anestesiología. Hospital Infantil de México «Federico Gómez». Ciudad de México.
\end{abstract}

Centro Médico Nacional «20 de Noviembre», ISSSTE. Ciudad de México. México.

\section{Correspondencia: Dr. Fabio Andrés Avellaneda-Suárez Anaxágoras 1004, Colonia Narvarte poniente, Delegación Benito Juárez, 03020, Ciudad de México, México. E-mail: fandres.sua@gmail.com}

Recibido: 29-09-2020 Aceptado: 20-11-2020

\section{INTRODUCCIÓN}

L as malformaciones broncopulmonares congénitas reúnen un grupo heterogéneo de alteración del desarrollo pulmonar durante la embriogénesis del pulmón y la vía aérea. El tipo de lesión histológica de presentación dependerá de varios factores como la edad gestacional, el nivel del árbol traqueobronquial en el que se produce la alteración en la embriogénesis, entre otros ${ }^{(1)}$.

La revisión realizada por Campos ${ }^{(2)}$ muestra que la incidencia anual de malformaciones pulmonares congénitas se estima en 56/100,000 recién nacidos vivos y de 30-42/100,000 habitantes si hablamos de la población general ${ }^{(1)}$. La tos y disnea son los síntomas de presentación más frecuentes (hasta en $60 \%)^{(2,3)}$. Las consideraciones anestésicas en el paciente pediátrico se basan en la inmadurez de sus órganos y sistemas, trastornos congénitos asociados, respuesta variable y, en ocasiones, los efectos de las drogas anestésicas y limitaciones al uso de altas concentraciones inspiradas de oxígeno ${ }^{(2,3)}$.

El examen riguroso de los ruidos cardíacos en búsqueda de soplos o arritmias y el examen del tórax nos permite ver la masa muscular, si es necesario se deberá auscultar el tórax, de manera intraoperatoria, en búsqueda de broncoespasmo para tratamiento del mismo, o bien se deberá evitar su exacerbación. La radiografía de tórax anteroposterior y lateral deberán revisarse haciendo énfasis en los hallazgos que pueden influenciar nuestro manejo anestésico, y si se cuenta con una tomografía axial computarizada (TAC) en necesario 
examinarla para observar la anatomía intratorácica (posición de la tráquea, influencia de tumores mediastinales sobre la vía aérea, etcétera ${ }^{(4)}$ ).

En cuanto a la técnica anestésica adecuada se deben considerar los aspectos como un buen control de la vía aérea, manejo del dolor perioperatorio, así como un destete precoz de la ventilación mecánica y una adecuada recuperación. Para el control del dolor postoperatorio hay dos opciones para niños menores de un año: la epidural caudal con altos volúmenes de anestésico local (ropivacaína al 2\%, 1.25 a 1.5 $\mathrm{mL} / \mathrm{kg}$ de peso) asociado siempre con morfina de 30 a $50 \mu \mathrm{g} /$ kg de peso en una sola punción prequirúrgica ${ }^{(4)}$. La otra gran alternativa, sobre todo para los neonatos, es la realización de una anestesia subaracnoidea con morfina de 10 a $15 \mu \mathrm{g}$ como dosis total con efectos analgésicos que persisten entre 24 y 48 horas con una sola punción ${ }^{(4)}$. Por lo tanto, y en las recomendaciones generales, se decide como una técnica anestésica ideal para este grupo etario, con patologías pulmonares y su pronta recuperación, la anestesia combinada, haciendo referencia a ésta como bloqueo caudal versus subaracnoideo y general balanceada.

\section{PRESENTACIÓN DEL CASO}

Se presenta el caso de un paciente masculino de 26 días de vida extrauterina, con diagnóstico preoperatorio de tumoración sólida vascularizada en base pulmonar izquierda, con indicación por grupo quirúrgico de realización de toracotomía posterolateral izquierda más lobectomía inferior izquierda y colocación de catéter venoso central.

Dentro de los antecedentes del recién nacido destaca: parto por vía abdominal por antecedente de polihidramnios severo, el cual se realizó bajo bloqueo epidural, obteniendo un producto, el cual no respira ni llora al nacer, con requerimiento de dos ciclos de ventilación con presión positiva, APGAR 6-8, Silverman Anderson 1-3, peso 2,800 gramos, talla $48 \mathrm{~cm}$, 37.5 semanas de gestación, con un peso adecuado para la edad gestacional, con posterior diagnóstico de taquipnea transitoria del recién nacido, pasa a Unidad de Cuidados Intensivos Neonatales (UCIN), originario de San Luis Potosí donde requiere manejo con presión positiva continua de las vías respiratorias (CPAP, por sus siglas en inglés) por 96 horas y manejo con fototerapia durante 36 horas por hiperbilirrubinemia multifactorial, se hace el diagnóstico ecocardiográfico de hipertensión pulmonar leve secundaria ( $35 \mathrm{mmHg}$ estenosis fisiológica de rama pulmonar izquierda) manejada con sildenafil $1 \mathrm{mg} / \mathrm{kg}$. A pesar de manejo, persiste con datos de dificultad respiratoria, por lo cual se toman estudios de gabinete: se observa tumoración vascularizada sólida en la base del pulmón izquierdo. Prueba COVID-19 negativa. Es remitido al Centro Médico Nacional «20 de Noviembre» para valoración y manejo de la patología descrita.
Es valorado por el Servicio de Cirugía Pediátrica, quienes deciden necesariamente llevar a cabo procedimiento quirúrgico, ingresa a sala de cirugía, con ventilación espontánea, activo, reactivo, con peso de $3.5 \mathrm{~kg}$, talla: $48 \mathrm{~cm}$. Se realiza monitorización tipo I, registrando los siguientes signos vitales: TA: 90/60 mmHg, FC: 135 latidos por minuto, FR: 38 respiraciones por minuto, $\mathrm{SatO}_{2}: 94 \%$, T: $36.1{ }^{\circ} \mathrm{C}$. Se decide dar una técnica anestésica combinada, previa preoxigenación con mascarilla facial a cinco litros por minutos, durante cinco minutos, inducción con fentanyl $15 \mu \mathrm{g} \mathrm{IV}$, propofol $10 \mathrm{mg}$ $\mathrm{IV}$, cisatracurio $500 \mu \mathrm{g} \mathrm{IV}$, se realiza laringoscopía indirecta atraumática, videolaringoscopio con hoja 0 Miller, se intuba con tubo endotraqueal número 3.5 al primer intento, sin globo, se fija a $9.5 \mathrm{~cm}$ de la comisura labial; se conecta a ventilador mecánico con los siguientes parámetros de volumen control: $\mathrm{FiO}_{2}: 100 \%$ flujo cuatro litros por minuto, Pmáx: 40, PINS: 25, FR: 45, TI: 1:1, PEEP: 0.

Posterior a esto, se ubica paciente en decúbito lateral izquierdo, se localiza hiato sacro y, previa sepsia y antisepsia, se introduce aguja número 24 en espacio epidural, se administra dosis de anestésico local con bupivacaína $12.5 \mathrm{mg}$ (2.5 mL) más morfina $100 \mu \mathrm{g}$ epidural, volumen total: $4.8 \mathrm{~mL}$, procedimiento realizado sin complicaciones. El mantenimiento se realiza con: sevoflurano a 0.8-1.0 Vol\% (concentración alveolar mínima 0.8), en total fentanyl $40 \mu \mathrm{g}$ IV fraccionado, tasa total de fentanyl $3.8 \mu \mathrm{g} / \mathrm{kg} / \mathrm{h}$. Oxígeno a dos litros por minuto, por sonda orotraqueal. Transanestésico: paciente hemodinámicamente estable durante el tras anestésico con FC: 112-130 latidos por minuto, FR: 45-50 respiraciones por minuto, TA: 90/60-100/70 mmHg, $\mathrm{SatO}_{2}: 95-98 \%$, acoplado a máquina de anestesia con los parámetros predichos. Se administró como adyuvantes: dexametasona $1.5 \mathrm{mg}$ IV.

$\mathrm{Al}$ término de la cirugía se traslada paciente bajo intubación orotraqueal, con bolsa máscara reservorio en ventilación asistida de tres litros por minuto, con FC: 120 latidos por minuto, TA: 78/53 mmHg, FR: 45 respiraciones por minuto, $\mathrm{SatO}_{2}: 96 \%$, escala de COMFORT: 10 , escala de CRIES (llanto, requerimiento de $\mathrm{O}_{2}$, incremento de los signos vitales, expresión facial y sueño/vigilia): 0 , un traslado a Unidad de Cuidados Intensivos Pediátricos (UCIP). El paciente se extubó 12 horas después del evento quirúrgico, y a las 24 horas se inició la vía oral con adecuada tolerancia, sin requerimientos de medicación analgésica de rescate, escala de CRIES: 0. El paciente es egresado siete días después del procedimiento con reporte de patología de malformación congénita de la vía aérea tipo 1 (Stocker) con abundantes linfangiectasias.

\section{DISCUSIÓN}

Las malformaciones congénitas de la vía aérea son patologías poco comunes presentes en el neonato y que pueden ser identificados desde la vida intrauterina hasta meses o años 
posteriores al nacimiento, teniendo una presentación bastante variable desde pacientes asintomáticos hasta algunos con datos francos de dificultad respiratoria desde su nacimiento o con presentación meses o años posteriores al mismo, lo cual supone variables a considerar para identificar el mejor método anestésico para su manejo quirúrgico.

Los desórdenes congénitos pulmonares tienen incidencia de entre 1:25,000-1:35,000 recién nacidos, usualmente un lóbulo completo del pulmón es reemplazado por una pieza de tejido pulmonar quístico no funcional ${ }^{(5)}$. La malformación surge por un desarrollo embrionario pulmonar anormal, específicamente por la detención en la fase pseudoglandular (semana 7-17 gestación), generando hiperplasia compensatoria de las vías aéreas distales con reemplazo del parénquima pulmonar normal por quistes, de tamaños y distribución variable ${ }^{(6)}$; existen dos clasificaciones por las cuales podemos identificar las malformaciones descritas, una introducida en 1997 por Stocker y colegas, la cual clasifica las lesiones en cinco tipos según su origen: tipo 0 (originado de tráquea o tejido bronquial), tipo I (originado de bronquios distales o bronquiolos proximales), tipo II y tipo III (origen acinar), tipo IV (origen acinar-alveolar) ${ }^{(7)}$. La otra clasificación basada en los hallazgos patológicos es la de Spencer, la cual diferencia tres tipos: I (ausencia completa de tejido pulmonar y bronquios), II (presencia de bronquios rudimentarios y ausencia de tejido pulmonar), III (pobre desarrollo de bronquios y pobre desarrollo de tejido pulmonar $)^{(8)}$. El diagnóstico y manejo de estas patologías dependerán principalmente de la sintomatología, el momento de aparición de la misma y el grado de compromiso pulmonar que se evidencien en los estudios diagnósticos.

Podemos observar que la mayoría de estos casos son identificados en neonatos e infantes y en la actualidad, gracias al uso del ultrasonido, se han podido observar desde la vida prenatal (semana 13-20 de gestación). El diagnóstico postnatal está asociado a la presencia de comorbilidades asociadas como falla cardíaca, hipertensión pulmonar, infecciones respiratorias a repetición, signos de dificultad respiratoria, etcétera, lo cual llevaría dentro del esquema diagnóstico a la realización de imágenes (radiografías de tórax, TAC de tórax), las cuales revelarían la patología pulmonar con un alto grado de fidelidad $^{(9)}$.

En cuanto al manejo y consideraciones anestésicas en este tipo de casos varía según la edad del paciente, comorbilidades asociadas, técnica quirúrgica aplicada y grado o extensión de la lesión, llevándonos a escenarios anestésicos complejos y demandantes, donde las metas anestésicas para este tipo de cirugía y de paciente son: prevenir la hipoxia, acidosis, hipercapnia (la cual puede incrementar las resistencias vasculares pulmonares), prevenir la reducción de las resistencias vasculares sistémicas y la depresión miocárdica asociada al uso de agentes anestésicos ${ }^{(9)}$.
La evaluación previa del paciente pediátrico va encaminada a los antecedentes perinatales, familiares y patológicos, un adecuado examen físico que debe ser completo pero dirigido al sistema cardiorrespiratorio, contemplando la vía aérea que pudiera ser potencialmente difícil (el contexto de una asociación con la tumoración), lo cual obligaría a diseñar un plan de manejo previo para el abordaje de la misma.

En nuestro caso se presenta el neonato programado para la realización de toracotomía más lobectomía izquierda, en el cual realizamos una técnica de ventilación pulmonar protectora bipulmonar (volumen tídal bajo y frecuencia respiratoria alta), la cual nos brindó una adecuada oxigenación y estabilidad durante el acto quirúrgico y anestésico, además no fue evidenciada una mayor dificultad en el abordaje quirúrgico, ya que fue un abordaje abierto.

En las recomendaciones para este tipo de pacientes se pueden usar dos técnicas de ventilación: la ventilación unipulmonar (vía endobronquial o con el uso de bloqueadores bronquiales) ${ }^{(10)}$ o la ventilación bipulmonar protectora, principalmente con volúmenes tídales bajos. El uso de una u otra técnica se deberá, principalmente, al tipo de técnica quirúrgica a usar, siendo más útil para el cirujano que prefiere la resección por toracoscopía el uso de la técnica unipulmonar, ya que expone mejor el campo quirúrgico y facilita la resección de la lesión ${ }^{(11)}$. Sin embargo, en la actualidad, se ha evidenciado que la técnica bipulmonar protectora en cirugía toracoscópica y/o abierta previene los casos de hipoxemia transoperatoria y sus consecuencias por la ventilación/perfusión inadecuada, además de los posibles errores en el posicionamiento de los bloqueadores bronquiales, por lo cual, aun cuando hay estudios que han mostrado disminución del número de complicaciones (infecciones, derrames pleurales, atelectasias) en pacientes sometidos a ventilación bipulmonar protectora durante el transanestésico en comparación con la ventilación unipulmonar, para elegir entre estas dos estrategias de ventilación se debe evaluar adecuadamente el tipo de cirugía que se va a practicar, además de la experticia y el conocimiento del anestesiólogo sobre estos dos métodos de ventilación transoperatoria ${ }^{(12)}$.

La técnica de inducción anestésica en nuestro caso se realizó bajo la combinación entre agentes inductores endovenosos (propofol) y agentes inhalados (sevoflurano), se ha observado que la inducción anestésica en este tipo de pacientes puede llevarse a cabo logrando una adecuada estabilidad hemodinámica, con el uso de anestésicos inhalados, inductores endovenosos o con la combinación de ambos ${ }^{(13)}$.

En este caso se decidió usar una inducción basada en anestésicos inhalados y endovenosos, logrando un buen plano anestésico y manteniendo las variables hemodinámicas sin mayores cambios; en cuanto al uso de bloqueadores neuromusculares, existe la recomendación de su uso momentos previos a la incisión quirúrgica, para poder mantener una ventilación controlada en el paciente previo a la incisión y 
disminuir el riesgo de neumotórax secundario a la presión positiva, sin embargo, es una decisión que debe valorar y planificar la técnica anestésica y ésta se debe escoger dependiendo si realmente el paciente tiene alto riesgo o no de presentar neumotórax secundario a la ventilación con presión positiva $^{(12)}$.

El mantenimiento anestésico durante el transanestésico fue basado en opioides (fentanilo IV) y agentes inhalados (sevoflurano) manteniendo CAM bajas, además se buscó brindar la mayor estabilidad hemodinámica durante el procedimiento. Asimismo, otra de las metas que se busca es la extubación temprana, lo que llevará a una adecuada evolución postoperatoria, que se puede valorar por la pronta tolerancia a la vía oral y alcanzar un adecuado nivel de analgesia ${ }^{(5,14,15)}$.

La técnica anestésica combinada en estos casos es la piedra angular para conseguir mejores desenlaces en este tipo de intervenciones ${ }^{(14)}$. Se ha propuesto para el manejo anestésico en este tipo de cirugías el uso de analgesia epidural, en este caso bloqueo caudal; se recomienda especialmente con el uso de catéteres epidurales para el control de analgesia durante el intraoperatorio y en el postoperatorio inmediato, esta recomendación ha demostrado tener mayor eficacia en el manejo analgésico con morfina en pacientes en edad pediátrica ${ }^{(15)}$.
Se puede ver un beneficio durante el transanestésico de menores concentraciones en los anestésicos inhalados, reducción en el uso de opioides, esto se observa con la extubación temprana, inicio temprano de la alimentación, adecuado control del dolor y reducción en los días de hospitalización ${ }^{(5,15)}$; en el caso descrito se usó la técnica anestesia combinada y anestesia general balanceada con bloqueo epidural (bloqueo caudal con bupivacaína $12.5 \mathrm{mg}$ [ $2.5 \mathrm{~mL}$ ] más morfina $100 \mu \mathrm{g}$ epidural, volumen total: $4.8 \mathrm{~mL}$ ) asegurando adecuadamente un nivel de analgesia perioperatoria y postoperatoria, logrando en nuestro paciente las metas anestésicas propuestas: se observó una rápida recuperación en la ventilación, reducción de analgésicos tanto opiáceos como no opiáceos, así como también reducción de los días de estancia hospitalaria que permitió un egreso temprano al procedimiento.

En resumen las malformaciones congénitas de la vía aérea son patologías que en su gran mayoría requieren una intervención quirúrgica, por lo cual se insinúa un gran reto tanto para los anestesiólogos generales como para los anestesiólogos pediatras, los cuales debemos entender que para un correcto manejo de este tipo de pacientes es necesario evaluar exhaustivamente el expediente clínico previo, la presencia de comorbilidades asociadas, el tipo de cirugía a realizar y plantear metas transoperatorias y postoperatorias claras para lograr mejores desenlaces en nuestros pacientes.

\section{REFERENCIAS}

1. Mondéjar López P, Sirvent Gómez J. Malformaciones pulmonares congénitas. Malacia y otras malformaciones congénitas de la vía aérea. Protoc diagn ter pediatr. 2017;1:273-297.

2. Campos Gustavo Julio. Anestesia en el paciente prematuro anestesiología en pediatría. Rev Mex Anestesiol. 2014;37:s92-s97.

3. Lam JC, Chui CH, Jacobsen AS, Tan AM, Joseph VT. When is a mediastinal mass critical in a child? Pediatr Surg Int. 2004;20:180-184.

4. Alcántara-Morales MA, García-Arreola DÁP. Toracoscopía en niños. Consideraciones anestésicas. Rev Mex Anestesiol. 2015;38:S104-S106.

5. Swapna AC, Nandini MD, Raylene JD, Madhu BG. Congenital pulmonary airway malformation with atrial septal defect and pulmonary hypertension for lobectomy-anesthetic considerations. Ann Card Anaesth. 2016;19:372-374.

6. Fromont-Hankard G, Philippe-homette P, Delezoide AL, Nessmann C, Aigrain Y, Peu-chmaur M. Glial cellderived neurotrophic factor expression in normal human lung and congenital cystic adenomatoid malformation. Arch Pathol Lab Med. 2002;126:432-436.

7. Mehta AA, Viswanathan N, Vasudevan AK, Paulose R, Abraham M. Congenital Cystic Adenomatoid Malformation: A Tertiary Care Hospital Experience. J Clin Diagn Res. 2016;10:SC01-SC04.

8. Zhang XT, Jin Z, Zhang Q, Li RM, Xu M, Babak F, et al. Congenital unilateral pulmonary malformation misdiagnosed as bronchial foreign body: a review of 14 cases. Acta Otolaryngol. 2010;130:971-976.
9. Fan D, Wu S, Wang R, Huang Y, Fu Y, Ai W, et al. Successfully treated congenital cystic adenomatoid malformation by open fetal surgery: A care-compliant case report of a 5-year follow-up and review of the literature. Medicine (Baltimore). 2017;96:e5865.

10. Arnaud Bonnard. Thoracoscopic Lobectomy for Congenital Pulmonary Airway Malformation: Where Are We in 2019? Eur J PediatrSurg. 2020;30:146-149.

11. Rajgire V, Tandale SR, Kelkar K, Band R. Neonate with congenital cystic adenoid malformation of lung for lobectomy: Anesthesia concerns. J Anaesthesiol Clin Pharmacol. 2018;34:561-562. doi: 10.4103/joacp. JOACP_393_17.

12. Narayanasamy S, Adler E, Mahmoud M, Burkley M, Lim FY, Subramanyam R. Airway management of congenital pulmonary airway malformation resection in neonates and infants: A case cohort study. Paediatr Anaesth. 2019;29:808-813.

13. Mohtar S, Hui TWC, Irwin MG. Anesthetic management of thoracoscopic resection of lung lesions in small children. Paediatr Anaesth. 2018;28:1035-1042.

14. Fajardo-Escolar AP, Díaz-Bohada L. Manejo anestésico en dos neonatos con malformación adenomatoide quística. Reporte de caso. Rev Colomb Anestesiol. 2017;45:76-80.

15. Guruswamy V, Roberts S, Arnold P, Potter F. Anaesthetic management of a neonate with congenital cyst adenoid malformation. Br J Anaesth. 2005;95:240-242. 